\title{
Vibron Excitations in Solid Hydrogen: A Generalized Binary Random Alloy Problem
}

\author{
J. L. Feldman, ${ }^{1,2}$ Jon H. Eggert, ${ }^{2}$ Jan De Kinder, ${ }^{3}$ Russell J. Hemley, ${ }^{2}$ Ho-kwang Mao, ${ }^{2}$ and D. Schoemaker ${ }^{3}$ \\ ${ }^{1}$ Complex Systems Theory Branch, Naval Research Laboratory, Washington, D.C. 20375-5345 \\ and George Mason University, Fairfax, Virginia 22030-4444 \\ ${ }^{2}$ Geophysical Laboratory and Center for High Pressure Research, Carnegie Institution of Washington, \\ 5251 Broad Branch Road, NW, Washington, DC 20015-1305 \\ ${ }^{3}$ Department of Physics, University of Antwerp (U.I.A.), B-2610 Wilrijk (Antwerp), Belgium
}

(Received 11 August 1994)

\begin{abstract}
Numerical calculations and Raman scattering measurements show that the vibrons in para-ortho mixtures of hydrogen are analogs of the one-electron states of a binary random alloy within the tightbinding Hamiltonian. Classical and Anderson-localized vibrons give important contributions to the spectral line shapes, which we demonstrate for a $\sim 20 \%$ ortho mixture close to the percolation limit at zero pressure. Based on a Hamiltonian in which the hopping term is constrained by independent measurements, the theory also accurately predicts the evolution of the vibron spectra under pressure to $15 \mathrm{GPa}$.

PACS numbers: 63.20.Hp, 62.50.+p, 63.20.Dj, 63.20.Ry
\end{abstract}

The role of structural and chemical disorder is of great importance to a number of fundamental and applied problems in condensed matter. For example, disorder in three dimensions produces mobility edges in one-electron energy spectra, a result which has profound effects on electron transport as a function of concentration. In two dimensions any amount of disorder produces localized states within a one-electron picture and on the basis of scaling theory [1]. Binary random alloys may yield both percolation- and Anderson-localization phenomena [2]. The nearest-neighbor single band tight-binding-like Hamiltonian with diagonal disorder is generally appealed to for describing these phenomena [3], and such an approach is not restricted to electronic properties [4]. However, this is a gross approximation for binary alloys, especially at high "defect" concentrations where it is expected that substitutional site disorder is not the only type of disorder present, and clustering of atom types will exist [3]. The single band nearest-neighbor tightbinding Hamiltonian is well known to be inappropriate for describing one-electron properties, and electron-electron interactions cannot be ignored [5]. In this Letter, we show that the same formalism used to describe electronic properties can be applied to localization phenomena involving vibrational excitations in molecular solids. In so doing, we identify the vibrons of solid hydrogen as a physical realization of the simplest binary random alloy model.

Solid hydrogen consists of para and ortho molecules (nuclear spin $I=1$ or 0 , respectively) randomly distributed on hexagonal close-packed sites for a substantial range of temperature and pressure [6-8]. At pressures of interest here, the molecules are nonrigid rotors, and at temperatures below $100 \mathrm{~K}$, essentially the only populated states are the $J=0$ and $J=1$ molecular rotational states, i.e., the ground states of para and ortho molecules, respectively, for $\mathrm{H}_{2}$. The rate of conversion between or- tho and para is sufficiently slow that the molecules can be assumed to be permanently $J=0$ or $J=1$ for the properties we shall discuss. Vibrons are the high-frequency vibrational excitations of the system $\left(\Omega_{v} \approx 4150 \mathrm{~cm}^{-1}\right.$ for solid $\mathrm{H}_{2}$ at ambient pressure). Van Kranendonk [8] first suggested that the vibrons in solid hydrogen are very well represented by a tight-binding-like Hamiltonian. A theory valid for small amounts of para or otho defects was applied to Raman intensities by James and Van Kranendonk (JK) [9]. In the JK model, the off-diagonal interactions are assumed to be independent of molecule type and diagonal disorder is present as a result of vibrationalrotational coupling for a single molecule [10].

The purpose of this paper is to show the correspondence between the vibron and electron problems in the tightbinding approximation, a parallel suggested by earlier work [9] but never examined in detail. We show this by measuring Raman spectra as a function of ortho-para concentration and pressure, and comparing the results with supercell calculations with the JK Hamiltonian. Although it is widely accepted that vibrational states in general can be localized, the spectral region localized for any particular model of disorder has been the subject of controversy [11,12]. The hydrogen vibron system corresponds to a band of high-frequency modes which, because they are optical modes, have the electronlike low- $k$ quadratic dispersion relation that is important for connecting the Ioffe-Regel criterion with localization [11]. It is this feature, in addition to the scalar nature of the dynamical variables (the intramolecular stretches), that gives the system its uniqueness in comparison to other vibrational problems [13]. Our results reveal the origin of a number of new and previously unexplained phenomena associated with vibron spectra measured under ambient and high-pressure conditions.

Recent studies [14] of the vibrons of hydrogen and deuterium have used the coherent potential approximation 
(CPA) method. It was shown that the model based on the JK Hamiltonian yielded an asymmetry in the Raman line shape in qualitative agreement with experiment at all concentrations. However, the CPA results show marked discrepancies with the experimental line shapes for concentrations of several percent or greater. In addition, the pressure dependences of the splitting and relative intensities of the $J=0$ and $J=1$ vibrons have been noted in previous work but have not been explained (see Ref. [7]). The latter is essential for understanding the character of the vibrons at very high pressures. We extend and test the JK model to address these issues.

High-resolution Raman measurements were performed on single crystals of $\mathrm{H}_{2}$ without applied pressure for a varying concentration of $\mathrm{o}-\mathrm{H}_{2}$. Research-grade gas was brought into contact with an Apachi gel catalyst at low temperatures to promote the $J=1$ to $J=0$ conversion [15]. The resulting gas was condensed into a He flow cryostat and the liquid was slowly cooled through the liquid-solid transition to produce a single crystal. Raman scattering of the lowest $\Delta J=2$ transitions allowed us to verify the $J=1$ concentration [16]. The light scattered from the sample was analyzed by a combination of a single pass Fabry-Pérot interferometer (finesse $F=38$ ) and a monochromator, which served as a broad bandpass filter, centered at the vibrational transition. Measurements were performed at $5 \mathrm{~K}$.

To calculate spectra from the theoretical model, we use the supercell method $[2,17]$. We use a cubic supercell of para and ortho molecules distributed randomly on a close-packed lattice with period boundary conditions [18]. The calculations are based on the JK Hamiltonian and JK assumption that the polarizability tensor is separable in the isolated molecule polarizabilities. The JK Hamiltonian can be written as

$$
H_{\mathrm{JK}}=\sum_{m} W_{m}^{\prime}|m\rangle\left\langle m\left|-\frac{1}{2} \sum_{m, n \neq m} \boldsymbol{\epsilon}_{m, n}^{\prime}\right| m\right\rangle\langle n| .
$$

Here $|m\rangle$ is the lowest vibrationally excited state on site $m, \epsilon_{m, n}^{\prime}$ is $0.5 \mathrm{~cm}^{-1}$ for nearest-neighbor molecules and zero otherwise, and $W_{m}^{\prime}$ is zero for ortho and $5.92 \mathrm{~cm}^{-1}$ for para molecules. We use these values of parameters for representing ambient pressure experiments. The eigenvalues $\omega_{j}$ and eigenvectors $e_{m, j}$ of a real symmetric matrix of dimension $N$ (the size of the supercell) determine the eigenvalues and eigenstates of $H_{\mathrm{JK}}$. In the $\mathrm{JK}$ model the polarizabilty is simply the sum of independent molecule terms, so the Raman intensity is proportional to

$$
R=\sum_{j}\left(\sum_{m} e_{m, j}\right)^{2} \delta\left(\omega_{j}-\omega\right) .
$$

This function of the eigenstates is obviously different from what enters the electronic conductivity (at $T=0 \mathrm{~K}$ ) where the electron diffusivity for states at the Fermi level is the relevant quantity.

The theoretical results are in excellent agreement with the Raman measurements, as shown for the para $\mathrm{H}_{2}$ tran-

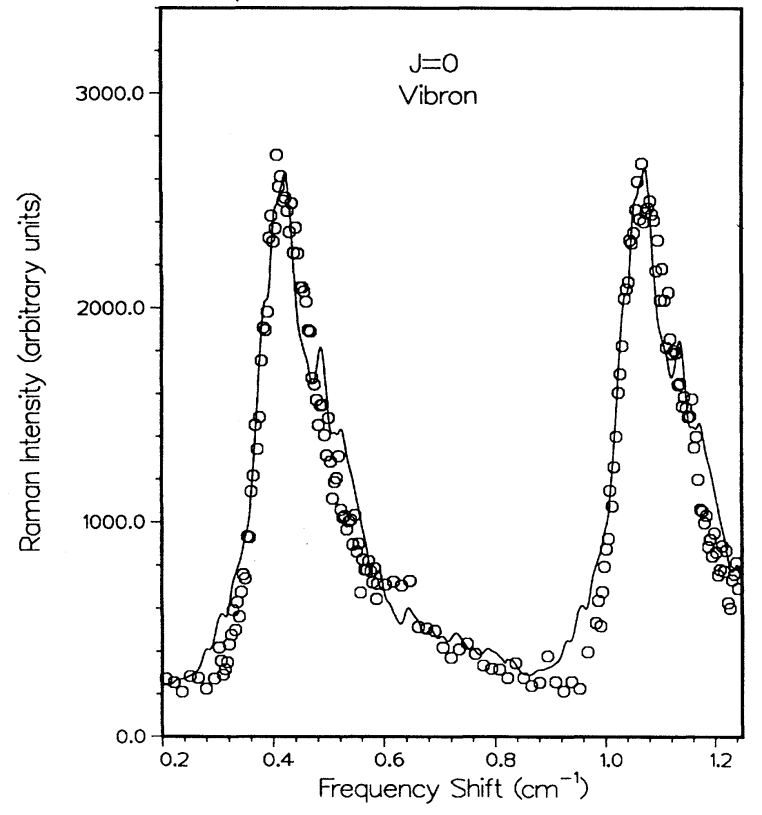

FIG. 1. Raman spectrum of the para peak for the $\sim 20 \%$ ortho sample. The peaks for different interference orders overlap slightly (see text). Calculations represent the average of 14 "runs" on a 5324 molecule supercell. The delta function in Eq. (2) is represented as a Lorentzian of width $0.01 \mathrm{~cm}^{-1}$. To compare theory and experiment, the energies were aligned, and the peak and background intensity (which was not measured) were adjusted.

sition in an $\left(\mathrm{o}-\mathrm{H}_{2}\right)_{0.19}\left(\mathrm{p}-\mathrm{H}_{2}\right)_{0.81}$ mixed crystal (Fig. 1). The theoretical "sample" here consisted of a 5324 molecule supercell with $20 \%$ ortho molecules and the average of 14 "runs." Since the Fabry-Pérot technique produces overlapping orders for the spectrum, we have superposed our calculational results using the periodicity determined in the experiment; the isolated peak, shown in the inset to Fig. 2, is seen to be somewhat broader and more asymmetric. We have also obtained excellent agreement between theory and experiment [19] for the ratio of intensities and differences in peak positions at other concentrations.

The analysis provides detailed information on the nature of the vibron states. To show this we calculate the inverse participation ratio $1 / p$,

$$
1 / p=\sum_{j} \sum_{m} e_{m, j}^{4} \delta\left(\omega_{j}-\omega\right) / \sum_{j} \delta\left(\omega_{j}-\omega\right),
$$

which has limiting values of 1 if the vibron is localized on a single molecule, and $1 / N$ if the vibron is equally partitioned among all the molecules. In Fig. 2 we superpose the vibron density of states $G$, the Raman cross section $R$, and $1 / p$ for our $20 \%$ ortho theoretical sample. The $J=1$ vibron band appears entirely localized (note that the ortho concentration is slightly below the percolation limit of the fcc lattice). The line shape of the Raman band is remarkably close to that obtained by Soots, Allin, and Welsh [19]. The inset highlights the vibrons under the 


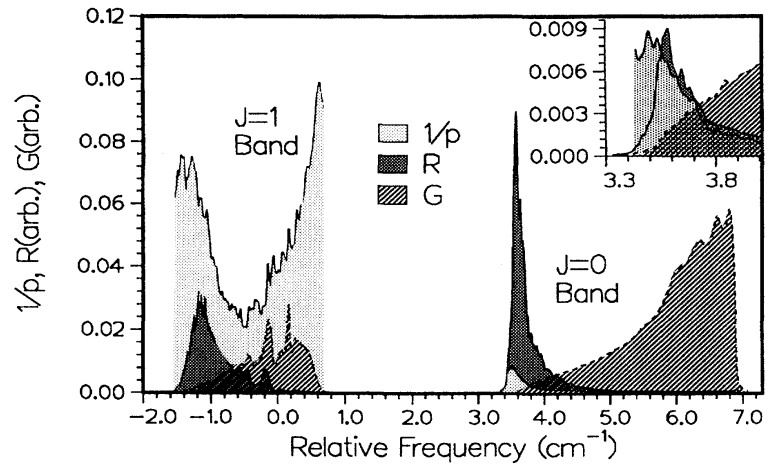

FIG. 2. Calculated inverse participation ratio $1 / p$, Raman intensity $R$, and vibron density of states $G$. The calculations were as in Fig. 1. The frequency is relative to the lowest vibrational energy excitation of an isolated ortho molecule (see text). Although the small fluctuations in the results are calculational noise, the larger amplitude structure (e.g., $G$ in the $J=1$ band) is characteristic of the model. The ratio of the area under the density of states curves for the $J=0$ and $J=1$ bands is 4 , which corresponds to the para-to-ortho concentration ratio. The lower portion of the $J=0$ band is shown in detail in the inset; $R$ and $G$ are rescaled.

$J=0$ Raman peak. $R$ is clearly large in the region of localized and fractal states and has a finite contribution at the mobility edge. The well-known tail in the density of states at the edge of the band is also obtained. We have also calculated $1 / p$ for smaller supercells to show that the edge states in the $J=0$ band are localized.

Variation of pressure provides both additional insight into these phenomena and a critical test of the theory. High-pressure experiments were performed by loading research-grade $\mathrm{H}_{2}$ in a modified Mao-Bell diamond anvil cell. Pressure was determined by standard ruby fluorescence techniques. We measured Raman spectra in a continuous-flow cryostat at $77 \mathrm{~K}$ using a $135^{\circ}$-scattering geometry. Our sample remained at $77 \mathrm{~K}$ long enough to allow an equilibrium ortho-para concentration of 5050. Our Raman system consisted of a spatial filter to limit stray-light scattering, a SPEX triplemate spectrometer with an 1800 grooves $/ \mathrm{mm}$ grating and a resolution of about $1 \mathrm{~cm}^{-1}$, and a liquid-nitrogen-cooled CCD detector. Representative Raman spectra as function of pressure are shown in Fig. 3.

Recent isotopic [20,21] and ir absorption studies [22,23] have shown that a bandwidth $B$ of the vibron in $\mathrm{H}_{2}$ increases rapidly with applied pressure from $3.0 \mathrm{~cm}^{-1}$ at ambient pressure [8] to $130 \mathrm{~cm}^{-1}$ at $36 \mathrm{GPa}$. From such studies we can determine the vibron bandwidth in $\mathrm{H}_{2}$ as a function of pressure. Least-squares fitting of the available data $[20,21]$ yields $B=3.162+4.098(P)^{1 / 2}+$ $2.868 P$, where $B\left(=6 \epsilon^{\prime}\right)$ is the energy difference in $\mathrm{cm}^{-1}$ between the origin and minimum of the band, and the pressure $P$ is in GPa. We have assumed $W$ to be pressure independent [24]. This approximation was motivated by the weak pressure dependence of the absolute position of the low frequency Raman peak.

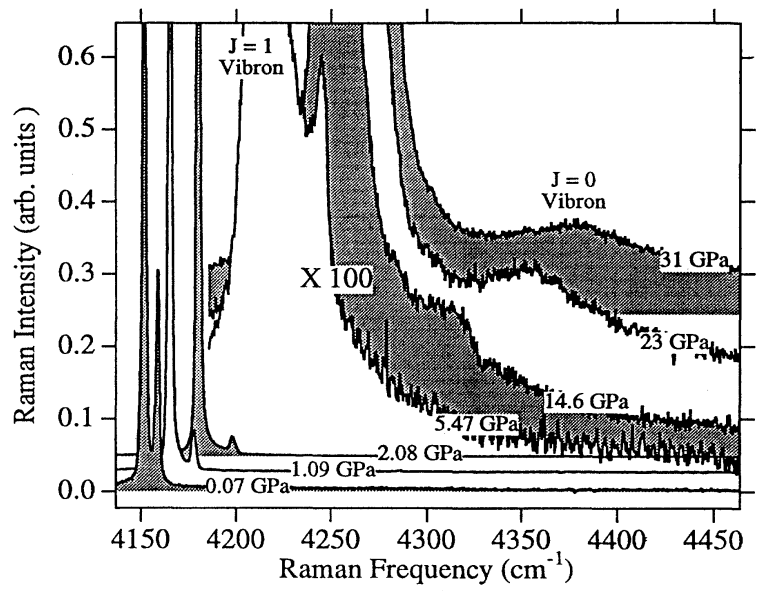

FIG. 3. Raman spectrum of solid hydrogen in the region of the intramolecular vibrons as a function of pressure from 0.07 to $31 \mathrm{GPa}$ at $77 \mathrm{~K}$.

In Fig. 4 we show the comparison between experiment and theory for the energy differences and integrated intensities using Eq. (3). The relative integrated intensities of the para and ortho peaks are seen to agree up to $15 \mathrm{GPa}$, or about fourfold compression of the solid [7]. Figure 4 shows the improvement in the match to experiment of the present results in relation to those of CPA calculations

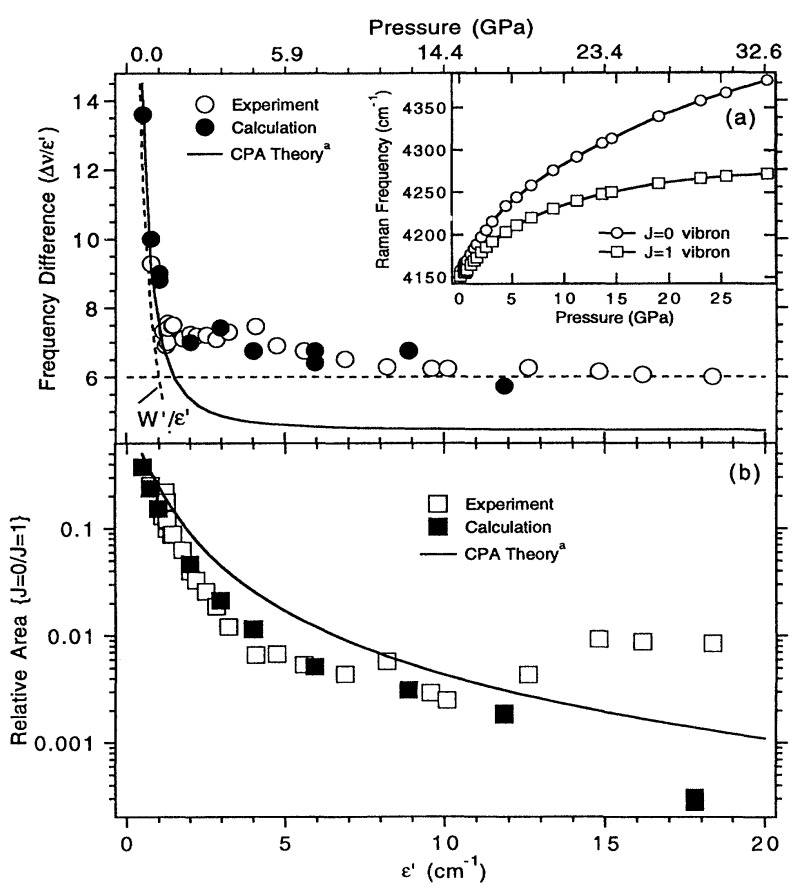

FIG. 4. (a) The difference in frequencies between the $J=0$ and $J=1$ peaks as a function of pressure. (b) The ratio of integrated intensities as a function of pressure. The CPA results shown were obtained through the approximate expressions, Eqs. (4.7), (4.8) and (4.10), of the first paper of Ref. [14]. See text for the $\epsilon^{\prime}$ vs pressure relation utilized here. 
[14]. The sudden change in the character of the curve for the frequency difference at $\epsilon^{\prime}=1 \mathrm{~cm}^{-1}$ occurs where the two bands $(J=1$ and $J=0)$ touch. Below that value the frequency difference is insensitive to pressure; above that point it is proportional to $\epsilon^{\prime}$. The calculations reveal a strong change in the character of the modes; in particular, excitations of both $J=0$ and $J=1$ molecules contribute to both peaks [25].

The theory thus accurately predicts a number of spectral properties of the vibrons over a range of conditions, despite the fact that no parameters in $H_{\mathrm{JK}}$ have been adjusted; all are determined independently from other measurements. In this regard, we note that recent experiments on deuterium [14] show a splitting of the (para) $J=$ 1 peak for a low para concentration sample. Although our preliminary results, using the $\mathrm{JK}$ parameters for deuterium, show a single $J=1$ peak, it is possible to obtain the splitting by adding an additional interaction between para molecules, which is reasonable to assume to be a quadrupole-quadrupole interaction [26]. A splitting similar to that observed experimentally was obtained provided we chose this contribution to $\epsilon^{\prime}$ to be $+0.1 \mathrm{~cm}^{-1}$. In addition, we have explored the effect of such a nonuniformity of $\epsilon^{\prime}$ on the larger concentration hydrogen results examined here as a function of pressure and believe that it contributes to the deviation seen between theory and experiment for the intensity ratios at pressures beyond $15 \mathrm{GPa}$ (Fig. 4). The results suggest increasingly complex interactions at these higher pressures [7].

In summary, we have obtained excellent agreement between Raman experiments on solid para-ortho hydrogen mixtures and theoretical calculations based on a tight binding Hamiltonian; calculations were performed by use of a supercell method that entailed periodic boundary conditions and random placements of ortho or para molecules on close-packed lattice sites. Our calculations strongly suggest that Anderson localization plays an important role in the Raman spectrum as a function of both concentration and pressure. An attractive feature of the approach is our ability to vary key variables that control the interactions, disorder, and thus localization, i.e., ortho-para concentration ( $J=0$ and $J=1$ molecules), pressure or density $\left(\epsilon^{\prime}\right)$, and isotopic species $\left(\mathrm{H}_{2}\right.$ vs $\left.\mathrm{D}_{2}\right)$. Variation of these parameters shows that it is possible to alter the nature of the Raman vibron excitations from localized to fractal to extended. Finally, we remark that mixed hydrogen and deuterium systems would provide an additional degree of disorder, i.e., a higher-order generalized alloy problem.

We are grateful to D. Huber, I. Mazin, R.E. Cohen, W. Vos, D. A. Papaconstantopoulos, B. Davidson, D. Singh, and J. Deppe for many useful discussions. The calculations were performed on the IBM 3090 at the Cornell supercomputing facility. This work was supported by the NSF (DMR-9304028) and NASA (NAGW1722). J.D.K. is a senior research assistant of the NFWO, Belgium.
[1] E. Abrahams, P.W. Anderson, D. C. Licciardello, and T. V. Ramakrishnan, Phys. Rev. Lett. 42, 673 (1979).

[2] J. M. Ziman, Models of Disorder (Cambridge University Press, Cambridge, 1979).

[3] H. Ehrenreich and L. M. Schwartz, Solid State Phys. 31, 150 (1976).

[4] R. J. Elliott, J. A. Krumhansl, and P. L. Leath, Rev. Mod. Phys. 46, 465 (1974).

[5] P. A. Lee and T. V. Ramakrishnan, Rev. Mod. Phys. 57, 287 (1985).

[6] I. F. Silvera, Rev. Mod. Phys. 52, 393 (1980).

[7] H. K. Mao and R. J. Hemley, Rev. Mod. Phys. 66, 671 (1994).

[8] J. Van Kranendonk, Physica (Utrecht) 25, 1080 (1959); J. Van Kranendonk, Solid Hydrogen (Plenum Press, New York, 1982).

[9] H. M. James, Phys. Rev. 164, 1153 (1967); H. M. James and J. Van Kranendonk, ibid. 164, 1159 (1967).

[10] J. L. Dunham, Phys. Rev. 41, 721 (1932).

[11] P. Sheng, M. Zhou, and Z.-Q. Zhang, Phys. Rev. Lett. 72, 234 (1994).

[12] A. Jagannathan, R. Orbach, and O. Entin-Wohlman, Phys. Rev. B 39, 13465 (1989).

[13] For a recent discussion of the character of vibrations in complex crystals, see P. B. Allen et al., Phys. Rev. B 49, 9073 (1994).

[14] J. De Kinder, A. Bouwen, D. Schoemaker, A. Boukahil, and D. L. Huber, Phys. Rev. B 49, 12754 (1994); 49, 12762 (1994)

[15] C. Sierens, A. Bouwen, E. Goovaerts, M. De Maziere, and D. Schoemaker, Phys. Rev. A 37, 4769 (1988).

[16] M. Leblans, A. Bouwen, C. Sierens, W. Joosen, E. Goovaerts, and D. Schoemaker, Phys. Rev. B 40, 6674 (1989).

[17] M. Schreiber, in Localization 1990, Proceedings of the International Conference on Localization, London, 1990, edited by K. A. Benedict and J. T. Chalker (IOP, Bristol, UK, 1991), p. 65, 1991; J. Non-Cryst. Solids 97, 221 (1987).

[18] Because we used a cubic supercell, a cubic closed packed rather than an hcp lattice was chosen; the energetics are identical for a nearest-neighbor interaction model (see Ref. [9]).

[19] V. Soots, E. J. Allin, and H. L. Welsh, Can. J. Phys. 43, 1985 (1965).

[20] D. M. Brown and W. B. Daniels, Phys. Rev. A 45, 6429 (1992).

[21] P. Loubeyre, R. LeToullec, and J. P. Pinceaux, Phys. Rev. B 45, 12844 (1992).

[22] M. Hanfland, R. J. Hemley, H. K. Mao, and G. P. Williams, Phys. Rev. Lett. 69, 1129 (1992).

[23] I. F. Silvera, S. J. Jeon, and H. E. Lorenzana, Phys. Rev. B 46, 5791 (1992).

[24] J.H. Eggert, H. K. Mao, and R.J. Hemley, Phys. Rev. Lett. 70, 2301 (1993).

[25] This will be elucidated in a future paper. For the purposes of the present study we use the standard convention of designating the two Raman bands as excitations of $J=0$ and $J=1$ molecules (i.e., ortho and para peaks).

[26] D. Huber (private communication). 